Trans

continentales
Transcontinentales

Sociétés, idéologies, système mondial

$1 \mid 2005$

Réforme et « Grand Moyen-Orient »

\title{
Histoire et modernité dans le monde arabe
}

Lucie RYZova, L'effendiyya ou la modernité contestée, Le Caire, Centre d'études et de documentation économiques et juridiques (CÉDÉJ), coll. « 15/20», 2004, 139 p.

\section{Édouard Méténier}

\section{OpenEdition}

\section{Journals}

\section{Édition électronique}

URL : http://journals.openedition.org/transcontinentales/1404

DOI : 10.4000/transcontinentales. 1404

ISBN : 978-2-8218-1410-3

ISSN : 1775-397X

Éditeur

Editions de la maison des sciences de l'homme

\section{Édition imprimée}

Date de publication : 31 décembre 2005

Pagination : 143-145

ISBN : 2200-92168-3

ISSN : 1950-1684

Référence électronique

Édouard Méténier, « Histoire et modernité dans le monde arabe », Transcontinentales [En ligne], 1 | 2005, document 11, mis en ligne le, consulté le 25 septembre 2020. URL : http://

journals.openedition.org/transcontinentales/1404; DOI : https://doi.org/10.4000/transcontinentales. 1404

Ce document a été généré automatiquement le 25 septembre 2020

Tous droits réservés 


\title{
Histoire et modernité dans le monde arabe
}

\author{
Lucie RYZOVA, L'effendiyya ou la modernité contestée, Le Caire, Centre \\ d'études et de documentation économiques et juridiques (CÉDÉJ), \\ coll. « 15/20», 2004, 139 p.
}

\section{Édouard Méténier}

\section{RÉFÉRENCE}

Lucie RYZOVA,L'effendiyya ou la modernité contestée, Le Caire, Centre d'études et de documentation économiques et juridiques (CÉDÉJ), coll. « 15/20 », 2004, 139 p.

1 Ce livre est le premier d'une nouvelle collection créée par le Centre d'études et de documentations économiques, juridiques et sociales du Caire, qui se donne pour objectif de promouvoir le travail de jeunes chercheurs œuvrant, à partir de problématiques originales informées par les sciences sociales, au décryptage des sociétés arabes contemporaines. Jeune chercheuse tchèque associée au CÉDÉJ, Lucie Ryzova a tiré d'un diplôme obtenu à Oxford en 2002 la base de l'ouvrage offert ici en français.

2 Le concept d'effendi occupe une place de choix dans toutes les descriptions et analyses des développements qu'a connus l'Égypte au cours du vingtième siècle sur les plans politique, social et culturel, notamment lorsqu'il s'agit de rendre compte de la naissance des mouvements nationalistes ou religieux radicaux. Ainsi, le terme effendiyya désigne généralement une sorte de classe moyenne composée des diplômés de l'enseignement général et supérieur; plus rarement, il signifie «lectorat" ou encore "classe bureaucratique ». Ce concept fréquemment employé, aux contours imprécis, est rarement défini ou explicité. Surexploité par les chercheurs, il n'est que peu étudié par eux. L. Ryzova s'attache donc à montrer que le terme ne peut pas être utilisé comme synonyme de "classe moyenne ", le groupe des effendis ne pouvant être considéré comme un substitut fonctionnel de celle-ci. S'appuyant sur des rapports produits dans 
les années 1940 par les ambassades britanniques du Moyen-Orient ainsi que sur l'étude de la presse populaire et sur des analyses d'œuvres littéraires ou cinématographiques d'époque, elle propose une lecture et une définition plus culturelles de l'effendiyya, mieux à même de rendre compte de sa réalité sociale : il s'agit en effet d'en dévoiler les contradictions internes ( $\mathrm{du}$ groupe, du terme) en partant du principe que ces dernières sont productives et cruciales si l'on veut parvenir à une intelligence des processus historiques dont elle fut l'agent et l'objet.

Dans la première partie de son ouvrage, L. Ryzova s'attache à définir l'effendiyya, à travers une approche historique. Elle distingue, entre la fin du XIX siècle et l'entre-deux guerres, trois générations d'effendis correspondant à autant de générations intellectuelles ayant chacune des assises socio-politiques et un projet politique différents, et qu'il convient de comprendre chacune dans leur rapport différentiel à l'État modernisateur. Elle centre ensuite son étude sur la troisième génération d'effendis, celle des jeunes hommes "fauteurs de troubles", qui émerge dans les années 1930-1940, et montre toute l'ambiguïté de sa situation sociale: principal revendicateur réclamant le "centre/espace médian " culturel et social, l'effendiyya de cette époque était néanmoins constamment et durement affectée par le changement. Plus que « la " classe moyenne, par ailleurs introuvable, elle en représentait "l'idée »: L. Ryzova appuie ici sa démonstration sur une étude fine des titres attribués dans les interactions sociales et de la manière dont ils fonctionnaient comme code de l'ordre sociétal. À partir de l'analyse d'itinéraires individuels, réels ou fictionnels, elle parvient ensuite à dresser un portrait très fin et tout en contraste de cette "génération d'hommes nouveaux ", au statut social et économique fort incertain, mais qui jouèrent un rôle fondamental dans les processus de changement politique que connut l'Égypte prénassérienne.

4 Dans la deuxième partie, elle s'intéresse à la manière dont, des années 1930 jusqu'au début des années 1950, le cinéma, comme instrument d'une nouvelle culture de masse, a constitué une source alternative de pouvoir tout en devenant le lieu sur lequel se sont déplacés les conflits sociaux. Ce faisant, le cinéma a largement contribué à conceptualiser l'effendi et à produire, à partir de cet idéal type, la représentation idéale de la classe moyenne égyptienne.

5 Ce livre est susceptible d'intéresser de nombreux chercheurs travaillant dans des contextes différents. Comme le révèlent les rapports britanniques consultés par L. Ryzova, la problématique de l'effendiyya concerne en effet tous les pays de l'Orient arabe, du Levant à la Mésopotamie. L'impact de la production culturelle et des mass média égyptiens (presse, radio, cinéma, etc.) dans l'ensemble du monde arabe, y compris le Maghreb et la péninsule Arabique, rend ce livre également pertinent pour les spécialistes de l'histoire sociale et culturelle de ces régions. Au-delà du cercle formé par les spécialistes du monde arabe, il convient de recommander la lecture de cet ouvrage aux chercheurs travaillant sur des espaces adjacents, comme la Turquie ou l'Iran, où pourraient se retrouver des développements comparables à ceux qui ont donné naissance à l'effendiyya étudiée en Égypte par L. Ryzova. Plus généralement, toutes les personnes travaillant sur le rapport entre émergence d'une culture de masse à travers le développement des mass média et production de représentations idéalisées d'icônes instituées de la classe moyenne trouveront dans ce livre de quoi nourrir leur réflexion.

6 L'intérêt même du livre et l'écho qu'il est susceptible de rencontrer bien au-delà du cercle des chercheurs travaillant habituellement sur l'Égypte nous conduisent 
cependant à avancer deux remarques. Sur le terrain formel, il subsiste quelques maladresses de traduction et de petites dissonances dans l'harmonisation des transcriptions de l'arabe. En ce qui concerne la bibliographie, essentiellement anglophone, ce qui se conçoit compte tenu du cadre dans lequel a été produit le travail à l'origine de cette publication, on peut déplorer l'absence de référence à un petit ouvrage dont l'intérêt est inversement proportionnel à la confidentialité de la diffusion qu'il a connue: Alain Roussillon, Réforme sociale et identité : essai sur l'émergence de l'intellectuel et du champ politique moderne en Égypte, Casablanca, Le Fennec, 1998. Cette remarque soulève une question plus vaste, celle de la juste place que la production académique francophone doit trouver sur le marché des références à partir desquelles se construit la recherche internationale en sciences sociales.

\section{AUTEURS}

\section{ÉDOUARD MÉTÉNIER}

Université de Provence-Institut de recherche sur le monde arabe et musulman (IREMAN) 